\title{
Optimal sizing of PV and battery-based energy storage in an off- grid nanogrid supplying batteries to a battery swapping station
}

\author{
Mingfei BAN $^{1,2}$ (1) Jilai YU ${ }^{1}$, Mohammad SHAHIDEHPOUR ${ }^{2}$, \\ Danyang GUO ${ }^{1}$
}

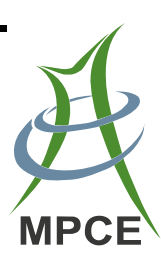

\begin{abstract}
Nanogrids are expected to play a significant role in managing the ever-increasing distributed renewable energy sources. If an off-grid nanogrid can supply fullycharged batteries to a battery swapping station (BSS) serving regional electric vehicles (EVs), it will help establish a structure for implementing renewable-energyto-vehicle systems. A capacity planning problem is formulated to determine the optimal sizing of photovoltaic (PV) generation and battery-based energy storage system (BESS) in such a nanogrid. The problem is formulated based on the mixed-integer linear programming (MILP) and then solved by a robust optimization approach. Flexible uncertainty sets are employed to adjust the conservativeness of the robust optimization, and Monte Carlo simulations are carried out to compare the performance of the solutions. Case studies demonstrate the merits of the proposed applications and verify our approach.
\end{abstract}

CrossCheck date: 17 May 2018

Received: 10 December 2017/Accepted: 17 May 2018/Published online: 5 September 2018

(C) The Author(s) 2018

$\triangle$ Mingfei BAN

mban2@iit.edu

Jilai YU

yupwrs@hit.edu.cn

Mohammad SHAHIDEHPOUR

ms@iit.edu

Danyang GUO

dg679@cornell.edu

1 Harbin Institute of Technology, Harbin 150001, China

2 Robert W. Galvin Center for Electricity Innovation, Illinois Institute of Technology, $10 \mathrm{~W}$ 35th Street \#1600, Chicago, IL, USA
Keywords Nanogrid, Battery swapping station, Electric vehicle, Renewable energy

\section{Introduction}

Nanogrids provide viable solutions for accommodating an ultra-high penetration level of distributed photovoltaic (PV) generation $[1,2]$. A PV-based nanogrid usually spreads out in a smaller geographic area and entails a smaller capacity. It is technically simpler than a microgrid $[3,4]$, but its implementation and operations are more flexible. A recent study established a definition for nanogrids and outlined their common grounds and differences with microgrids [5]. In principle, nanogrids represent smaller versions of microgrids [2], thus they are subject to fewer technical challenges and regulatory hurdles than microgrids [1]. Nanogrids also have the advantage of being perceived by investors as less risky than microgrids. Besides, the modular nature of nanogrids delivers an opportunity to connect them to form a microgrid. Thus, this paper restricts its presentation to PV-based nanogrids.

A battery-based energy storage system (BESS) [6] is indispensable for compensating for the imbalances between generation and demand in an off-grid nanogrid $[7,8]$. Nevertheless, a nanogrid employing a stand-alone BESS is very costly. Accordingly, studies focus on sharing generation and storage resources via transmission lines [9-11]. However, the cascading confluence of power electronics systems inevitably introduces new challenges, such as frequency and voltage transients, for PV-based nanogrids. Thus, our previous study [12] presents a system which allows sharing PV generation and energy storage resources by swapping batteries. It supposes that off-grid nanogrids could store surplus PV in batteries and then 
supply fully-charged batteries to a battery swapping station (BSS) serving electric vehicles (EVs). In this paper, we address a capacity planning framework for such a nanogrid. Various generation and storage technologies are feasible for deploying nanogrids [13, 14], this paper focuses on a PV plus BESS configuration.

At the planning stage, the uncertainties of PV generation and local demands should be adequately estimated and managed. Stochastic optimization (SO) [15] is a typical approach to cope with such uncertainties. It assumes prior probability distributions of uncertainties are known, and it could provide a probabilistic guarantee of its solutions $[16,17]$. The SO has been employed to solve optimal capacity planning problems for microgrids $[18,19]$ and EV charging stations [20, 21]. However, this approach has some practical limitations, since it involves the accurate prior probability distributions, and its solution process incorporates plenty of scenarios that require significant computational effort.

The robust optimization (RO) handles the uncertainties using only the range of uncertain data rather than detailed probability distributions. It has received extensive attention and has been successfully applied to microgrid planning $[22,23]$ and energy storage planning [24]. All these works indicate that RO is an effective method for ensuring system operation against the worst scenarios [25]. However, it is overly conservative to satisfy all scenarios, and decision makers typically prefer a tradeoff between robustness and economy in some practical applications. Therefore, it is attractive to investigate methods for reducing the conservativeness of the RO solutions.

Adjusting uncertainty sets is one strategy to reduce the conservativeness of RO solutions. Bertsimas and Sim [26] constructed uncertainty sets in RO problems and introduced the concept of uncertainty budgets $(\Gamma)$ to flexibly adjust the level of robustness of the solutions. An attractive aspect of their method is that it does not significantly increase the complexity of the original optimization problem. It has been widely adopted in solving various problems including unit commitment [27], generation expansion planning [28], load scheduling [29], and demand response [30], etc. This paper employs it for determining the PV and BESS mix in a nanogrid.

Main contributions are summarized and highlighted as:

1) Optimal sizing for an off-grid nanogrid is modeled in the MILP formulation and then solved with an RO approach, which minimizes the investment cost while guarantees the desired level of reliability in the energy supply.

2) The RO approach can control the robustness level of the capacity planning solutions by adjusting parameter $\Gamma$. It overcomes that if only the worst-case scenarios are utilized in the capacity planning, the results could be over-conservative.

3) Monte Carlo simulation-based analysis is introduced to emulate the long-term performance of the solutions, and case studies investigate the effects of battery swapping on optimal capacity planning for a nanogrid.

\section{Framework of a nanogrid-based BSS}

This section introduces the architecture of a typical PVBESS nanogrid and demonstrates its potentials for serving a BSS. Besides, the framework for a nanogrids-based BSS is briefly illustrated.

\subsection{Nanogrid}

Figure 1 presents the schematic of a hybrid AC/DC nanogrid, Keating Nanogrid, at the campus of the Illinois Institute of Technology (IIT) [1]. The nanogrid powers a sports center, and it is an important part within the campuswide IIT Microgrid [31]. Its PV arrays power the LED lighting systems directly, while simultaneously charge the batteries. It can work in both of off-grid mode and gridconnected mode. Successful operation of this nanogrid illustrates how a PV-plus-battery system can deliver resilient electricity to local users.

The peak load of the Keating Nanogrid is close to 150 $\mathrm{kW}$, whereas the installed capacity of its rooftop PV panels is $173.5 \mathrm{~kW}$. A BESS $(330.4 \mathrm{kWh})$ compensates the imbalances between PV generation and demand [1]. The BESS stores energy from periods of high PV output and uses it in periods of power shortage, and thus ensures reliable operation of the nanogrid. At certain hours that the BESS is fully charged, the nanogrid will curtail the surplus PV generation or feed it back to the broader IIT Microgrid.

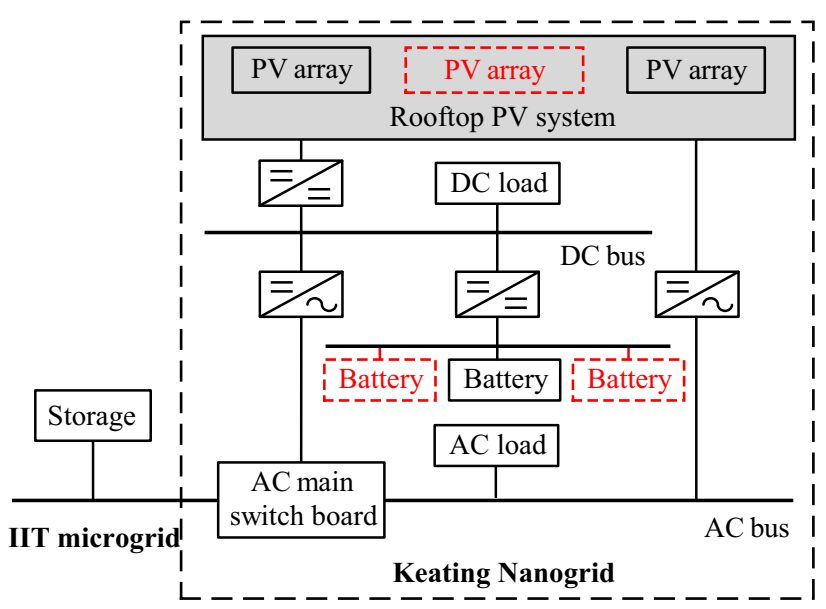

Fig. 1 Architecture of Keating Nanogrid within IIT microgrid 
Nevertheless, this means strict electricity quality restrictions and costly bi-directional interface capacity.

Supplying fully-charged batteries to a BSS is an alternative for handling the surplus PV generation. In this way, the Keating Nanogrid could provide enough energy to a BSS for serving 16 to 20 EVs [32]. Furthermore, if the nanogrid could install more PV panels and batteries, as shown in the red parts in Fig.1, it could contribute more energy storage to the BSS while enhancing itself with more resilience. Accordingly, a nanogrid-based BSS is a potential approach for implementing a PV-to-vehicle system and reinforcing the operation of nanogrids.

A modular interface (MI) is embedded in the nanogrid for facilitating battery swapping. As given in Fig. 2, the MI integrates a group of modular batteries, bidirectional converters, and a battery management system. The modular batteries can be seen as mobile energy storages [33] and substitute for conventional storage of a nanogrid. The MI is a generalized facility that is beneficial for scalability. Various types of nanogrids with distinct characteristics can supply batteries to the BSS, as long as they are equipped with such an MI. Besides, the MI ensures that the plug-out and plug-in of batteries have no influences on the nanogrid's local operation.

Since the nanogrid supplies fully-charged batteries to the BSS, a monotonic charge strategy [34] is adopted in the MI's battery management system. This strategy forces a consistent charging/discharging direction for each battery. When a battery has been monotonously fully-charged, it gets ready for being swapped or powering the nanogrid. By this means, it can make sure that there are enough fullycharged batteries for performing battery swapping operation.

\subsection{Nanogrid-based BSS}

At a BSS, depleted batteries in an EV can be swapped momentarily with fully-charged ones. And existing works for PV-based BSSs [32, 35] mainly focus on centralized structures, in which large-scale centralized PV generation recharges all the depleted batteries centrally. Such structures require a lot of space to install centralized PV panels,

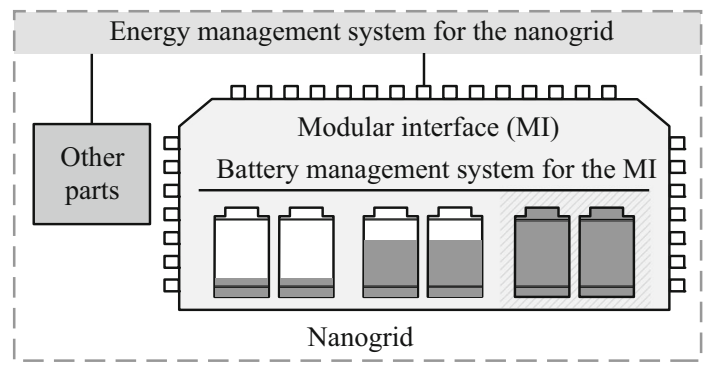

Fig. 2 Modular interface embedded in a nanogrid and thus they are costly when the BSSs locate in urban areas. We develop a nanogrid-based BSS framework for implementing a distributed-PV-to-vehicle system, as shown in Fig. 3. A nanogrid can store its surplus PV generation in batteries and then exported fully-charged batteries to the BSS via a delivery truck. The truck also delivers depleted batteries from the BSS to the nanogrid, where the depleted batteries are integrated into the MI and recharged.

There is an agreement between the nanogrid and the BSS. According to the agreement, the BSS bears a portion of investment costs for installing additional BESS in the nanogrid, while the nanogrid exports fully-charged batteries to the BSS. Consequently, the nanogrid achieves cost savings and the BSS benefits from a lower investment on PV panels. Moreover, the agreement states the total amount of the exported fully-charged batteries, rather than forces a specific amount for each sub period. Thus, it is flexible and beneficial for balancing the variable PV generation and local demands in the nanogrid. Hence, a nanogrid-based BSS framework catalyzes the popularity of electrified transportation [36] and also magnifies the benefits of nanogrids.

Note that there should be multiple nanogrids to implement a nanogrid-based BSS system. The nanogrids can be owned by an operator or different operators. This will influence the choice of the control strategy. For example, if one operator owns all the nanogrids, a centralized control approach is preferable. Otherwise, a hierarchical approach can be more appropriate. A comprehensive analysis should be conducted for determining the control strategy for the nanogrid-based BSS, which is beyond the scope of this paper.

\section{Problem formulation}

Strategic capacity planning is important in achieving optimal long-term performance for a nanogrid [21]. In this section, capacity planning for a single nanogrid is modeled in the MILP formulation. A well-performing nanogrid

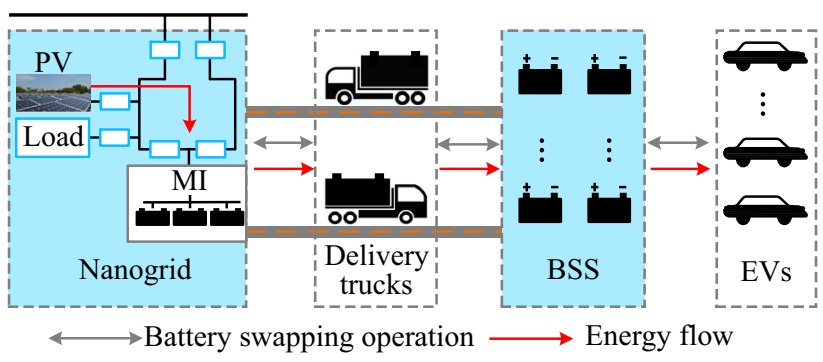

Fig. 3 Sketch for nanogrid-based BSS 
should be able to handle the majority of uncertainties without the utility grid [18], and thus we only consider the off-grid cases.

\subsection{Deterministic formulation}

The deterministic problem is formulated as follows:

Objective function: (1) minimizes the investment cost for PV generation and battery capacity. And $e^{\text {con }}$ denotes the amount that the BSS can bear in the total cost for the installing BESS.

Constraints: (2) represents the power balance and the PV outputs are constrained by (3). The discharging/charging variables for the battery are governed by the power limits (4) and (5), logical relations for status (6), hourly energy balance (7) and energy capacity limits (8)-(10). Equation (11) avoids end-of-horizon effects by setting the final energy storage level to be close to its initial value. For battery swapping, (12) represents hourly energy export capacity constraints, (13) avoids frequent but inefficient energy exports by stating the minimum interval between two sequential battery export operations. Equation (14) states the lower limits for the total amount of energy exported from the nanogrid to the BSS before time $t$ (referred to as battery swapping demand hereafter), which depends on the energy agreement. Equations (15) and (16) are bounds of decision variables. Related symbols in equations are defined in Table 1.

$\min C=I C_{\mathrm{PV}} e^{\mathrm{PV}}+I C_{\mathrm{ESS}}\left(e^{\mathrm{BESS}}-e^{\mathrm{con}}\right)$

s.t. $\quad P V(t)+P^{\mathrm{dis}}(t)=P L(t)+P^{\mathrm{ch}}(t)+c(t) \quad \forall t$

$0 \leq P V(t) \leq P V_{\mathrm{G}}(t) e^{\mathrm{PV}} \quad \forall t$

$0 \leq P^{\mathrm{dis}}(t) \leq I^{\mathrm{dis}}(t) \bar{P}^{\mathrm{dis}} \quad \forall t$

$0 \leq P^{\mathrm{ch}}(t) \leq I^{\mathrm{ch}}(t) \bar{P}^{\mathrm{ch}} \quad \forall t$

$I^{\mathrm{dis}}(t)+I^{\mathrm{ch}}(t) \leq 1 \quad \forall t$

$E(t)=E(t-1)+\eta^{\mathrm{ch}} P^{\mathrm{ch}}(t) \Delta t-\frac{P^{\mathrm{dic}}(t) \Delta t}{\eta^{\mathrm{dis}}}-E^{\mathrm{sw}}(t) \quad \forall t$

$\underline{E} \leq E(t) \leq \bar{E} \quad \forall t$

$\underline{E}=s^{*} e^{\mathrm{BESS}}$

$\bar{E}=S^{*} e^{\mathrm{BESS}}$

$E(0)-\Delta E \leq E(N T) \leq E(0)+\Delta E$

$I^{\mathrm{sw}}(t) \underline{E}^{\mathrm{sw}} \leq E^{\mathrm{sw}}(t) \leq I^{\mathrm{sw}}(t) \bar{E}^{\mathrm{sw}} \quad \forall t$

$\sum_{\tau=t}^{t+T^{\mathrm{sw}}}\left(1-I^{\mathrm{sw}}(\tau)\right) \geq T^{\mathrm{sw}} I^{\mathrm{sw}}(t) \quad \forall t$
Table 1 Nomenclature

\begin{tabular}{|c|c|}
\hline Parameter & Description \\
\hline$I C_{\mathrm{PV}}$ & Investment cost of $\mathrm{PV}$ units per size $(\$ / \mathrm{kW})$ \\
\hline$I C_{\mathrm{BESS}}$ & Investment cost of BESS units per size $(\$ / \mathrm{kWh})$ \\
\hline$C$ & Investment cost for the nanogrid $(\$)$ \\
\hline$e^{\mathrm{PV}}$ & PV panel installed capacity $(\mathrm{kW})$ \\
\hline$e^{\mathrm{BESS}}$ & BESS installed capacity (kWh) \\
\hline$e^{\text {con }}$ & $\begin{array}{l}\text { The amount that the BSS can bear in the total cost for } \\
\text { installing BESS }(\mathrm{kWh})\end{array}$ \\
\hline$P V(t)$ & PV output at time $t(\mathrm{~kW})$ \\
\hline$P^{\mathrm{dis}}(t)$ & Discharging power of BESS at time $t(\mathrm{~kW})$ \\
\hline$P^{\mathrm{ch}}(t)$ & Charging power of BESS at time $t(\mathrm{~kW})$ \\
\hline$P L(t)$ & Local demand of nanogrid at time $t(\mathrm{~kW})$ \\
\hline$c(t)$ & Curtailment of PV generation at time $t(\mathrm{~kW})$ \\
\hline$P V_{\mathrm{G}}(t)$ & Normalized maximum PV generation rate $(\mathrm{kW} / \mathrm{kW})$ \\
\hline $\begin{array}{l}I^{\mathrm{dis}}(t) / \\
\quad I^{\mathrm{ch}}(t)\end{array}$ & Binary status variable for BESS charging/discharging \\
\hline$E(t)$ & Stored energy in the BESS at time $t(\mathrm{kWh})$ \\
\hline$\eta^{\mathrm{dis}} / \eta^{\mathrm{ch}}$ & Discharging/charging efficiencies of the BESS (\%) \\
\hline$s^{*} / S^{*}$ & Lower/upper limits for stored energy in the BESS $(\mathrm{kWh})$ \\
\hline$E^{\mathrm{sw}}(t)$ & Exported energy by battery swapping at time $t(\mathrm{kWh})$ \\
\hline$I^{\mathrm{sw}}(t)$ & Binary status variable for battery swapping operation \\
\hline$\kappa^{\mathrm{ex}}(t)$ & $\begin{array}{l}\text { Lower limit for total exported energy from the nanogrid } \\
\text { to the BSS before time } t(\mathrm{kWh})\end{array}$ \\
\hline$t$ & Index for time (hour) \\
\hline$N T$ & Total number of periods under study ( 24 hours) \\
\hline$\Delta t$ & Unit time interval which is one hour in this paper (hour) \\
\hline$T^{\mathrm{sw}}$ & $\begin{array}{l}\text { Minimum interval between two sequential battery } \\
\text { swapping operations for the nanogrid (hour) }\end{array}$ \\
\hline$(\bar{g})$ & Upper limit for item $(\bullet)$ \\
\hline$(\underline{g})$ & Lower limit for item $(\bullet)$ \\
\hline
\end{tabular}

$\sum_{\tau=1}^{t} E^{\mathrm{sw}}(\tau) \geq \kappa^{\mathrm{ex}}(t) \quad \forall t$

$\underline{e}^{\mathrm{PV}} \leq e^{\mathrm{PV}} \leq \bar{e}^{\mathrm{PV}}$

$\underline{e}^{\mathrm{BESS}} \leq e^{\mathrm{BESS}} \leq \bar{e}^{\mathrm{BESS}}$

Note that we take $e^{\text {con }}$ as a predefined parameter in the above formulations. An alternative is taking $e^{\text {con }}$ in (1) as a variable, and then (14) can be revised as:

$\sum_{\tau=1}^{t} E^{\mathrm{sw}}(\tau) \geq \vartheta^{\mathrm{ex}}(t) e^{\mathrm{con}} \quad \forall t$

where $\vartheta^{\mathrm{ex}}(t)$ is a predefined ratio stating that the total amount of exported energy at time $t$ should be not less than $\vartheta^{\mathrm{ex}}(t) e^{\mathrm{con}}$. More related discussions are given in Section 4 . 


\subsection{Robust formulation}

For the deterministic problem described in (1)-(16), hourly PV generation $P V(t)$ and local demand $P L(t)$ are uncertain parameters. It is a practical problem to determine the optimal PV and BESS mix that minimizes the investment cost while guarantees a required level of robustness against the uncertainties. RO has potentials for tackling with such problems. Classical RO method minimizes the total cost in the worst cases, and it solves the following generic min-max problem with objective function $f(\bullet)$ and constraints $g(\bullet)$.

$\min _{\boldsymbol{x} \in \boldsymbol{X}} \max _{\boldsymbol{h} \in \boldsymbol{H}} \boldsymbol{f}(\boldsymbol{x}, \boldsymbol{h})$

s.t. $\quad \boldsymbol{g}(\boldsymbol{x}, \boldsymbol{h}) \leq \mathbf{0}$

where $\boldsymbol{x}$ is a vector of decision variables belonging to set $\boldsymbol{X}$; $\boldsymbol{h}$ represents the uncertain parameters taking values in the uncertainty set $\boldsymbol{H}$.

However, the above method sometimes tends to be too conservative. To tackle this problem, [26] proposed an adjustable RO method to handle the problem (20) with polyhedral uncertainty sets.

$\left\{\begin{array}{l}\min \boldsymbol{c}^{\prime} \boldsymbol{x} \\ \text { s.t. } \sum_{n=1} \hat{a}_{m n} x_{n} \leq b_{m} \\ l_{n} \leq x_{n} \leq u_{n}\end{array}\right.$

where uncertain coefficient $\hat{a}_{m n}$ takes values in interval $\left[a_{m n}-\Delta a_{m n}, a_{m n}+\Delta a_{m n}\right]$, in which $\Delta a_{m n}$ is the maximum deviation from the nominal value $a_{m n}$. The corresponding polyhedral uncertainty sets are described as follows:

$\hat{a}_{m n}=a_{m n}+\xi_{m n} \Delta a_{m n}\left|\sum_{m=1}\right| \xi_{m n}\left|\leq \boldsymbol{\Gamma}_{m},\right| \xi_{m n} \mid \leq 1, \quad \forall m, n$

where $\xi_{m n}=\left(\hat{a}_{m n}-a_{m n}\right) / \Delta a_{m n}$, which obeys an asymmetric distribution that is described using only the upper and the lower uncertainty boundaries.

For (20), its robust counterpart formulation with consideration of conservatism is recast as an MILP formulation in (22).

$$
\left\{\begin{array}{lll}
\min & \boldsymbol{c}^{\prime} \boldsymbol{x} & \\
\text { s.t. } & \sum_{n=1} \hat{a}_{m n} x_{n}-z_{m} \boldsymbol{\Gamma}_{m}-\sum_{n=1} p_{m n} \leq b_{m} & \forall m \\
& z_{m}+p_{m n} \geq \Delta a_{m n} x_{n} & \forall m, n \in J_{m} \\
& z_{m}+p_{m n} \geq-\Delta a_{m n} x_{n} & \forall m, n \in J_{m} \\
& p_{m n} \geq 0 & \forall m, n \in J_{m} \\
& z_{m} \geq 0 & \forall m \in J_{m} \\
& l_{n} \leq x_{n} \leq u_{n} & \forall n
\end{array}\right.
$$

For constraint $m$, a parameter $\Gamma_{m}$ taking values in the interval $\left[0,\left|J_{m}\right|\right]$ (where $J_{m}$ is the set of coefficients $\hat{a}_{m n}, n \in$
$\left.J_{m}\right)$ is used to adjust the robustness of the solution. $z_{m}$ is the dual variable of constraint $m$ and $p_{m n}$ is an auxiliary variable to linearize the model. By varying $\Gamma_{m}$, we have the flexibility of adjusting the robustness of the solutions. If $\Gamma_{m}$ $=0$, no deviations are considered, and thus the constraints are same as that of the nominal problem. Otherwise, if up to $\left\lfloor\Gamma_{m}\right\rfloor$ of the $\left|J_{m}\right|$ coefficients change within their bounds, and up to one coefficient changes by $\left(\Gamma_{m}-\left\lfloor\Gamma_{m}\right\rfloor\right) \Delta a_{m n}$, then the solution of (22) will always remain feasible. Thus, if $\Gamma_{m}$ is $\left|J_{m}\right|$, the maximum deviation is considered, and this is the most conservative case. In other words, predefined $\Gamma_{m}$ can control the tradeoff between the robustness and economic performance of the solutions, and thus we call $\Gamma_{m}$ as uncertainty budgets. The detailed description of this adjustable RO method can be found in [26].

According to (22), the robust counterparts for the deterministic formulation are established as follows:

$$
\begin{aligned}
& P V(t)-p v(t)-s_{\mathrm{PV}} \boldsymbol{\Gamma}_{\mathrm{PV}}+P^{\mathrm{dis}}(t) \\
& \quad=P L(t)-p l(t)-s_{\mathrm{LD}} \boldsymbol{\Gamma}_{\mathrm{LD}}+P^{\mathrm{ch}}(t)+c(t) \\
& p v(t)+s_{\mathrm{PV}} \geq\left(\overline{P V}_{\mathrm{G}}(t)-\underline{P V}_{\mathrm{G}}(t)\right) e_{\mathrm{PV}} \\
& p l(t)+s_{\mathrm{LD}} \geq \overline{P L}(t)-\underline{P L}(t)
\end{aligned}
$$

where $\Gamma_{\mathrm{PV}}$ and $\Gamma_{\mathrm{LD}}$ are predefined uncertainty budgets, which adjust the conservativeness of the solutions; $p l(t)$ and $p v(t)$ are dual variables of constraints (2) and (3); $s_{\mathrm{PV}}$ and $s_{\mathrm{LD}}$ are auxiliary variables used for linearization purpose. Equation (23) forces the auxiliary variables to take values following the uncertainty budgets. If uncertainty budgets are equal to 0 , then (23) is identical to the deterministic formulation in (2). Otherwise, the new formulation will force increments in the installed PV and BESS capacity to seek higher robustness of the solutions.

\subsection{Solution methodology}

The proposed robust formulation for the optimal capacity planning has been transformed into an MILP formulation. The MILP is a deterministic optimization technique that can be solved by well-developed commercial solvers, such as CPLEX. General advantages of the MILP method include: 1) it can guarantee the global optimality of the solution; 2) it provides a more direct measure of optimality; 3) it is flexible and can enhance modeling capabilities and adaptability [37]. Poor performance in scalability is the main disadvantage of the MILP. However, it is not an issue in this paper since our problem has a small size. There is extensive literature comparing the pros and cons of MILP with its competitors [38, 39].

Figure 4 outlines the solution procedure. The required input data include PV generation, local demands, and battery swapping demand, etc. 


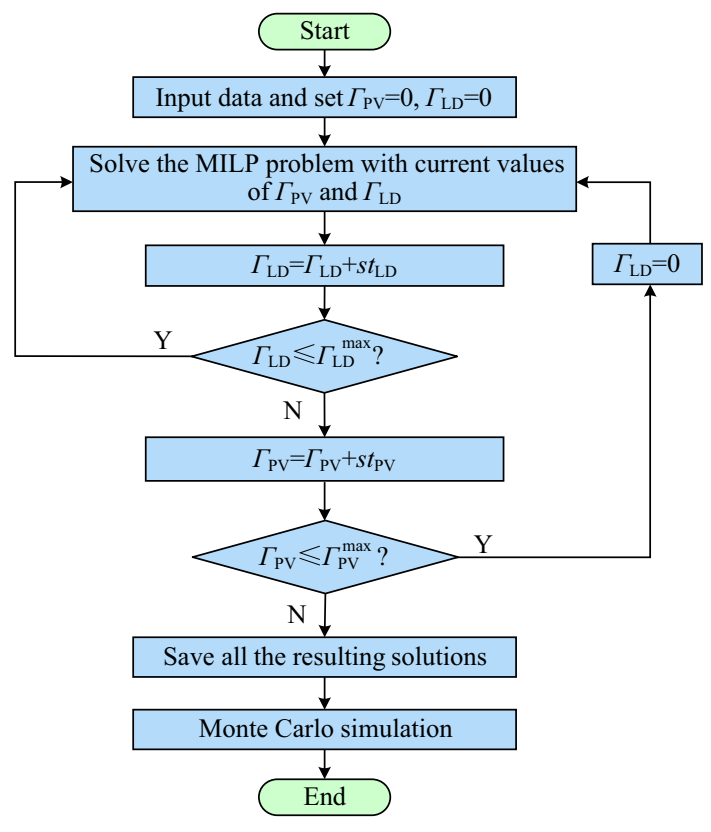

Fig. 4 Flowchart for the solution methodology

Step 1: Based on measurements and historical data, define upper and lower bounds for uncertain parameters. Set all uncertainty budgets $\left(\Gamma_{\mathrm{PV}}\right.$ and $\left.\Gamma_{\mathrm{LD}}\right)$ to zero, which corresponds to the least conservative case

Step 2: Solve the MILP with current values of the uncertainty budgets, and save the corresponding solutions

Step 3: Increase the uncertainty budget $\Gamma_{\mathrm{LD}}$ with step size $s t_{\mathrm{LD}}$. Then, if the current value of $\Gamma_{\mathrm{LD}}$ is not larger than its upper limit $\Gamma_{\mathrm{LD}}^{\max }$, go to Step 2, otherwise, increase the uncertainty budget $\Gamma_{\mathrm{PV}}$ with step size $s t_{\mathrm{PV}}$. If $\Gamma_{\mathrm{PV}}$ is larger than its upper limit $\Gamma_{\mathrm{PV}}^{\max }$, go to Step 4 , otherwise, reset $\Gamma_{\mathrm{LD}}$ to zero and go back to Step 2. Note that the values of $s t_{\mathrm{LD}}$ and $s t_{\mathrm{PV}}$ result in a tradeoff between desired accuracy and computational performance. In theory, for $N$ uncertain parameters and each $\Gamma_{\tau}$ increases from $\Gamma_{\tau}^{\min }$ to $\Gamma_{\tau}^{\max }$ in $s t_{\tau}$ increments, the total number of combinations to be solved is $\prod_{\tau=1}^{N}\left[s t_{\tau}^{-1}\left(\Gamma_{\tau}^{\max }-\Gamma_{\tau}^{\min }\right)+1\right]$

Step 4: Save the solutions for different values of the uncertainty budgets, and then run all of them against the Monte Carlo scenarios to identify their performance. The Monte Carlo simulation calculates infeasible samples and the infeasibility probability regarding different realizations of the uncertain parameters. Lower infeasibility probability means the solution is more robust against uncertainties and can cover more cases.
Then it can give a measurement of how much the decision maker can rely on each solution

\section{Simulation and results}

Figures 5 and 6 show the profiles for the involved hourly PV generation and local demands. The input data were measured at a nanogrid at the IIT campus [1]. Data measured in January and June are selected to represent the seasonal variations, and an hourly averaging was performed based on these two months to limit the number of variables. Then the minimum and maximum of the hourly averaged values are set as lower and upper bounds of uncertain parameters.

The investment costs for PV and BESS are $\$ 839 / \mathrm{kW}$ and $\$ 945 / \mathrm{kWh}$, respectively. Charging and discharging efficiencies are 0.9. Upper and lower energy limits for the battery are 0.2 and 0.8 . Besides, the initial value of stored energy in the batteries is $60 \%$, and the final value should fall in an interval of 55\%-65\%. The lower limits for the total amount of exported energy $\kappa^{\mathrm{ex}}(t)$ at 13:00, 18:00 and 24:00 are $340 \mathrm{kWh}, 700 \mathrm{kWh}$ and $750 \mathrm{kWh}$, respectively.

\subsection{Solutions for capacity planning}

We give capacity planning solutions for the nanogrid. Figure 7 presents a benchmark for the case that the nanogrid exports no fully-charged batteries to a BSS (Case 1), whereas Fig. 8 shows the results for the proposed framework (Case 2). Case 1 and Case 2 show that we have to

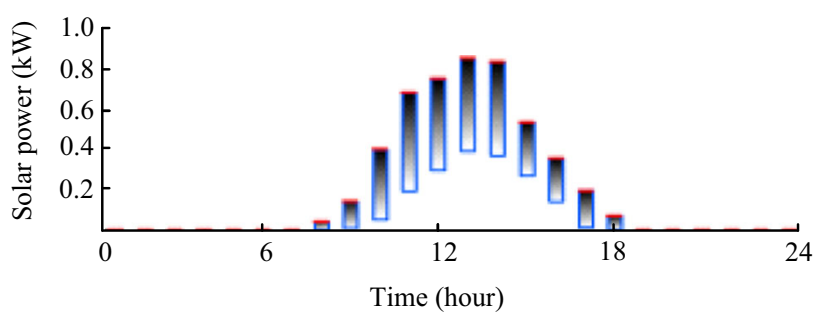

Fig. 5 Profiles for daily solar power, where the red line represents the maximum value and the box spans the range

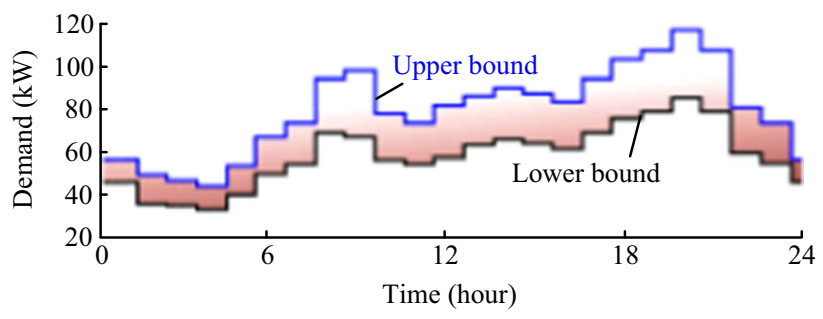

Fig. 6 Profiles for daily local demands in the nanogrid 

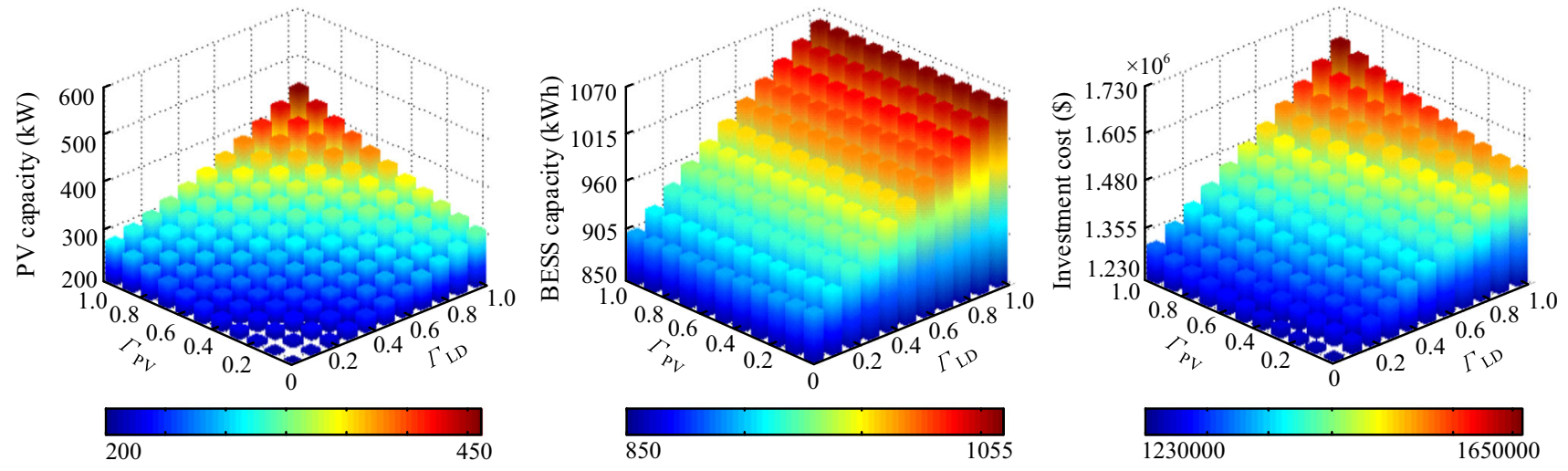

Fig. 7 Installation of PV, BESS and investment versus different uncertainty budgets $\Gamma_{\mathrm{PV}}$ and $\Gamma_{\mathrm{LD}}$ in Case 1 (the nanogrid exports no batteries to a BSS)
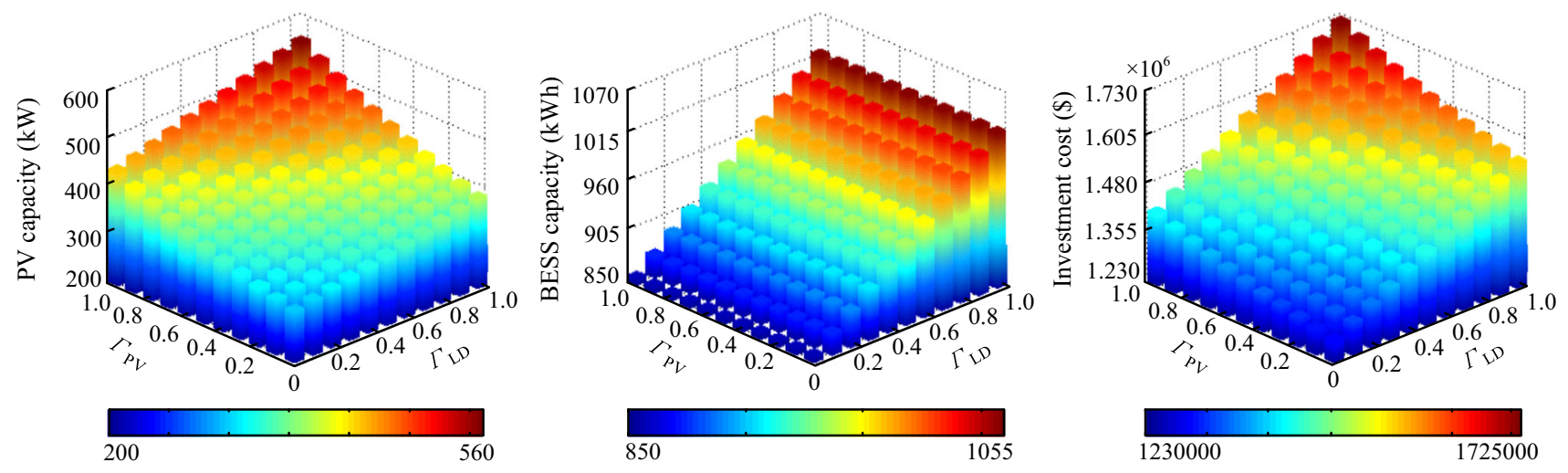

Fig. 8 Installation of PV, BESS and investment versus different uncertainty budgets $\Gamma_{\mathrm{PV}}$ and $\Gamma_{\mathrm{LD}}$ in Case 2 (the nanogrid exports batteries to a BSS)

install more PV panels and batteries for seeking higher reliability. In Case 2, as the nanogrid supply energy to both of its local load and a BSS, it has to install more PV panels $(25 \%-51 \%)$ than that in Case 1. However, the installation of batteries in Case 2 is 3\%-5.5\% less than that in Case 1, even though that the nanogrid in Case 2 exports batteries to a BSS. This is because that the increased PV generation alleviate the burden of energy storage in the nanogrid.

The investment cost in Fig. 8 has no consideration of the agreement that the BSS bears a portion (defined as $\delta$ ) of the investment costs for BESS. If the BSS can bear $10 \%$ of the cost for BESS, the total investment in Case 2 will fall below that in Case 1, as given in Table 2. Table 2 also compares Cases 1 and 2 adopting different values of $\delta, \Gamma_{\mathrm{PV}}$ and $\Gamma_{\mathrm{LD}}$. It shows that the nanogrid will achieve significant cost savings in Case 2. If the BSS can pay for all the BESS, the nanogrid can save more than half of the total investment. Note that this is also beneficial to the BSS, since it avoids installing a large number of PV panels.

Case 2 takes $e^{\text {con }}$ as a predefined value, however, as remarked earlier, another alternative is taking $e^{\text {con }}$ as a variable. Case 3 takes $e^{\text {con }}$ as a variable, and the lower limits for total exported energy $\left(\vartheta^{\mathrm{ex}}(t) e^{\mathrm{con}}\right)$ before 13:00, 18:00 and 24:00 are defined as 35\%,75\% and $95 \%$ of $e^{\text {con }}$, respectively. Then Table 3 lists the results. It demonstrates that when the nanogrid seeks higher reliability, it will make a higher energy agreement with the BSS. Besides, taking $e^{\text {con }}$ as a variable further enhances the flexibility in the sizing PV and BESS in a nanogrid, and thus, Case 3 shows better performance than Case 2 .

Here, we re-define the lower limits for total exported energy before 15:00, 18:00 and 24:00 as $45 \%, 75 \%$ and $90 \%$ of $e^{\text {con }}$, respectively. Then the resulting counterparts for Table 3 are given in Table 4. It demonstrates that different profiles of battery swapping demand from the BSS may result different sizes of a nanogrid.

\subsection{Performance of the solutions}

The Monte Carlo simulation generates a large number of scenarios to represent uncertainties in hourly PV generation and local demand. And the number of studied 
Table 2 Investment cost for Cases 1 and 2 with different $\delta$

\begin{tabular}{|c|c|c|c|c|c|c|}
\hline \multirow[t]{2}{*}{$\overline{\Gamma_{\mathrm{PV}}}$} & \multirow[t]{2}{*}{$\Gamma_{\mathrm{LD}}$} & \multirow[t]{2}{*}{ Cost for Case $1\left(10^{6} \$\right)$} & \multicolumn{4}{|c|}{ Cost for Case $2\left(10^{6} \$\right)$} \\
\hline & & & $\delta=0$ & $\delta=10 \%$ & $\delta=50 \%$ & $\delta=100 \%$ \\
\hline 0 & 0 & 1.2395 & 1.2886 & 1.2022 & 0.8566 & 0.4246 \\
\hline 0.2 & 0.2 & 1.3084 & 1.3623 & 1.2759 & 0.9303 & 0.4983 \\
\hline 0.4 & 0.4 & 1.3826 & 1.4401 & 1.3537 & 1.0081 & 0.5761 \\
\hline 0.6 & 0.6 & 1.4637 & 1.5254 & 1.4390 & 1.0934 & 0.6614 \\
\hline 0.8 & 0.8 & 1.5207 & 1.6173 & 1.5309 & 1.1853 & 0.7533 \\
\hline 1.0 & 1.0 & 1.6577 & 1.7186 & 1.6322 & 1.2866 & 0.8546 \\
\hline
\end{tabular}

Table 3 Installation of PV, BESS and investment cost in Case 3

\begin{tabular}{lllcll}
\hline$\Gamma_{\mathrm{PV}}$ & $\Gamma_{\mathrm{LD}}$ & Installation of PV $(\mathrm{kW})$ & Installation of BESS $(\mathrm{kWh})$ & Values of $e^{\text {con }}(\mathrm{kWh})$ & Investment cost $\left(10^{6} \$\right)$ \\
\hline 0 & 0 & 307.5 & 858.3 & 780.3 & 0.3888 \\
0.2 & 0.2 & 349.1 & 890.0 & 809.1 & 0.4326 \\
0.4 & 0.4 & 396.5 & 921.6 & 837.8 & 0.4819 \\
0.6 & 0.6 & 450.7 & 953.2 & 866.6 & 0.5379 \\
0.8 & 0.8 & 513.6 & 984.8 & 895.9 & 0.6023 \\
1.0 & 1.0 & 587.2 & 1164.8 & 924.1 & 0.6772
\end{tabular}

Table 4 Installation of PV, BESS and investment cost in Case 3

\begin{tabular}{lllcll}
\hline$\Gamma_{\mathrm{PV}}$ & $\Gamma_{\mathrm{LD}}$ & Installation of PV $(\mathrm{kW})$ & Installation of BESS $(\mathrm{kWh})$ & Values of $e^{\text {con }}(\mathrm{kWh})$ & Investment cost $\left(10^{6} \$\right)$ \\
\hline 0 & 0 & 301.3 & 859.9 & 781.7 & 0.3829 \\
0.2 & 0.2 & 342.3 & 891.5 & 810.5 & 0.4261 \\
0.4 & 0.4 & 388.9 & 923.2 & 839.3 & 0.4747 \\
0.6 & 0.6 & 442.4 & 954.9 & 868.1 & 0.5300 \\
0.8 & 0.8 & 504.2 & 986.6 & 896.9 & 0.5934 \\
1.0 & 1.0 & 576.7 & 1018.3 & 925.7 & 0.6672 \\
\hline
\end{tabular}

scenarios is reduced by a fast-forward technique [40]. Consequently, 900 scenarios are employed, and we run the resulting solutions in Cases 1 and 2 against these scenarios to assess their performance. The simulated PV generation and local demand scenarios follow a normal distribution with a standard deviation (volatility) of $10 \%$ of expected values, which are median value in Figs. 6 and 7 . Note that when $\delta=10 \%$, Case 2 has similar investment cost with Case 1 (see Table 2). Thus, Case 2 with $\delta=10 \%$ is taking as an example to be compared with Case 1 .

Figure 9 shows the proportion of infeasible hours that the nanogrid suffers a stored energy level below $20 \%$, which is denoted as an infeasible probability (IP). It also provides the amount of averaged curtailed PV generation (CPV). In both of Case 1 and Case 2, as expected, the number of infeasible hours tends to decrease as the
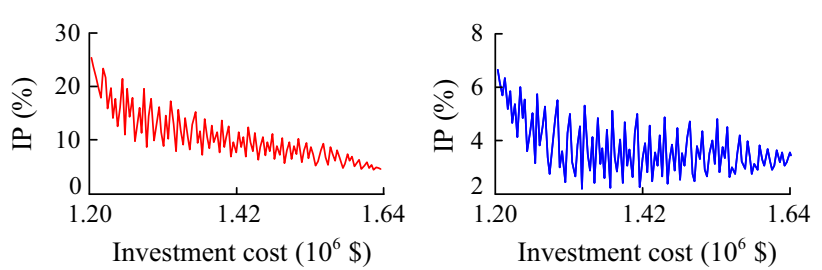

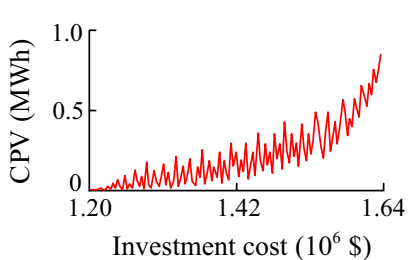

(a) Case 1

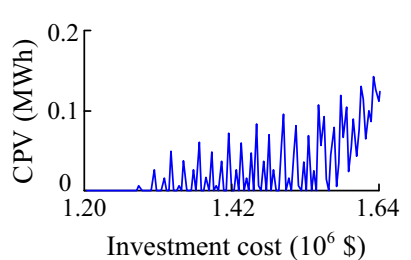

(b) Case 2
Fig. 9 Average IP and CPV versus the investment cost in Cases 1 and 2 
investment cost increases, while the amount of CPV follows an opposite trend. Table 5 provides a more detailed analysis.

Table 5 and Fig. 9 show that Case 2 exhibits better performance than Case 1. At similar investment cost level, Case 2 has lower IP and less CPV. Providing energy storage as service to the BSS can enhance the economy and reliability of the nanogrid. Thus the proposed structure realizes the potential benefits of dispersed batteries that are used for backup energy storage in the nanogrids and also catalyzes the electrification of the public transport $[15,41]$.

Table 6 briefly lists results derived from the classical SO [15] and RO method [23]. Table 6 also gives the results of deterministic optimization (DO) using the median value in Figs. 6 and 7. In general, it also shows that Case 2 performs better than Case 1. Besides, the RO solution is the most expensive one. Correspondingly, the RO solution performs quite well in the feasibility analysis as it is feasible in almost all scenarios. However, this also indicates that the uncertainty the RO solution can withstand is somehow much larger than the practical requirement. The SO approach seems to be less conservative than the worstcase-oriented RO approach, and it is barely better than the deterministic one.

Comparison between Tables 5 and 6 further illustrates the advantages of the proposed approach. It can control the robustness of its solutions. Moreover, by adjusting uncertainty budgets, it can provide much more options than the classical SO and RO approaches. This meets the require- ments of decision makers preferring a tradeoff between robustness and economy in practical applications.

\subsection{Energy dispatch with one group of solutions}

Figure 10 demonstrates daily power components of the nanogrid in Case 2 . Here we take capacity planning solution for $\Gamma_{\mathrm{PV}}=0.6$ and $\Gamma_{\mathrm{LD}}=0.6$ as example, and it consists of $41.1 \%$ PV capacity increment, $11.7 \%$ battery capacity

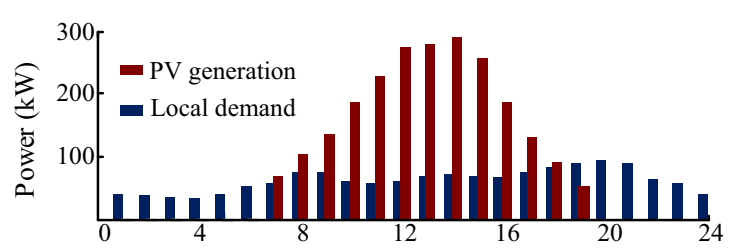

Time (hour)
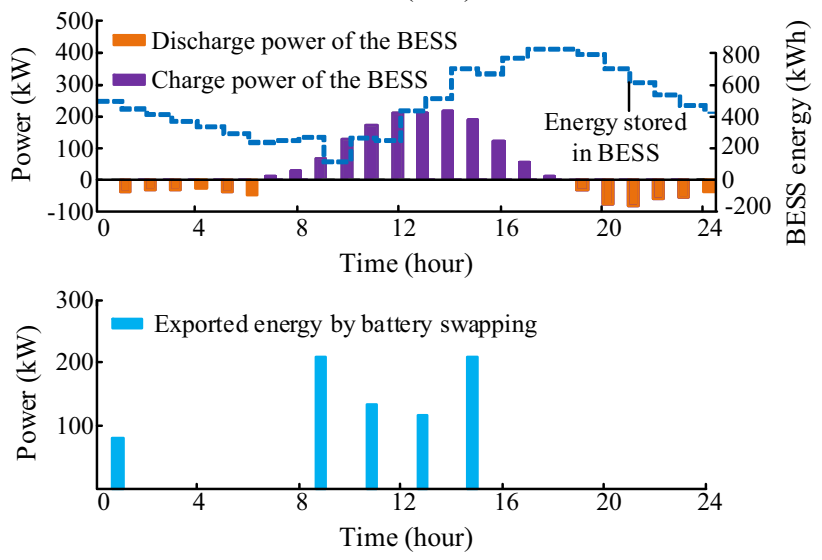

Fig. 10 Demonstration of daily power components of the nanogrid

Table 5 IP and CPV versus uncertainty budgets in Cases 1 and 2

\begin{tabular}{llllll}
\hline$\Gamma_{\mathrm{PV}}$ & $\Gamma_{\mathrm{LD}}$ & $\mathrm{IP}(\%)$ & & \multicolumn{2}{c}{$\mathrm{CPV}(\mathrm{kWh})$} \\
\cline { 3 - 5 } & & Case 1 & Case 2 & Case 1 \\
\hline 0 & 0 & 25.4 & 6.7 & 0 & 0 \\
0.2 & 0.2 & 17.8 & 5.0 & 12.2 & 79.4 \\
0.4 & 0.4 & 11.6 & 3.7 & 236.0 & 0 \\
0.6 & 0.6 & 7.9 & 3.1 & 353.2 & 53.0 \\
0.8 & 0.8 & 6.8 & 3.1 & 847.0 & 75.5 \\
1.0 & 1.0 & 4.5 & 1.6 & 127.6 \\
\hline
\end{tabular}

Table 6 Comparisons between different solution methods

\begin{tabular}{|c|c|c|c|c|c|c|}
\hline \multirow[t]{2}{*}{ Solution method } & \multicolumn{2}{|l|}{ IP $(\%)$} & \multicolumn{2}{|c|}{ CPV (kWh) } & \multicolumn{2}{|c|}{ Cost $\left(10^{6} \$\right)$} \\
\hline & Case 1 & Case 2 & Case 1 & Case 2 & Case 1 & Case 2 \\
\hline DO & 16.3 & 5.3 & 213.1 & 69.9 & 1.4583 & 1.3758 \\
\hline SO & 13.2 & 3.9 & 198.5 & 57.6 & 1.5773 & 1.3927 \\
\hline RO & 0.4 & 0.2 & 836.6 & 152.5 & 1.6656 & 1.6401 \\
\hline
\end{tabular}



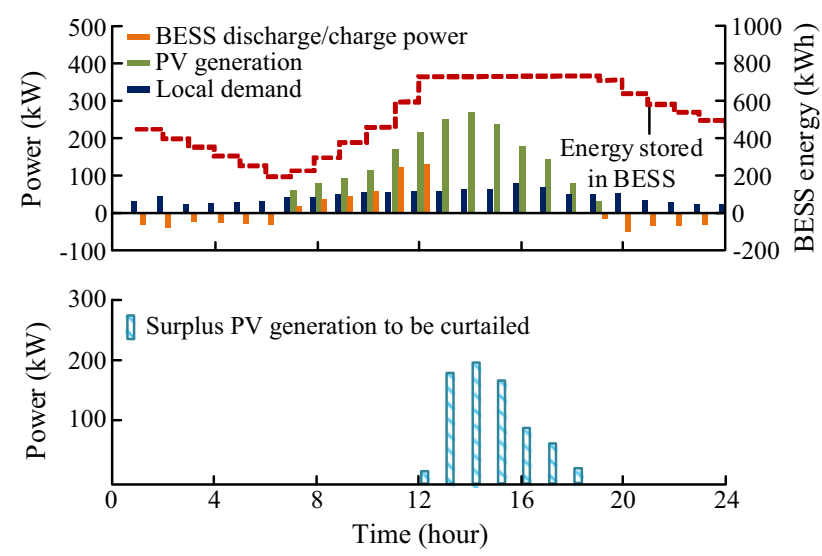

Fig. 11 Demonstration of daily power components of the nanogrid without exporting batteries to the BSS

increment, and $18.4 \%$ total investment increment than the base case $\left(\Gamma_{\mathrm{PV}}=0\right.$ and $\left.\Gamma_{\mathrm{LD}}=0\right)$.

The nanogrid keeps charging between Hour 7 and Hour 18. Since it can export fully-charged batteries (at Hours 9, 11,13 and 15) to the BSS, its BESS always has enough capacity to store the surplus PV generation. Thus, no PV generation is curtailed. At Hour 16, the nanogrid stops exporting batteries to prepare for the periods with insufficient PV generation. The nanogrid periodically adjusts its energy dispatch to meet local energy requirements and to fulfillment energy exporting agreement with the BSS. As mentioned in Sect. 2, although the agreement claims that the nanogrid should export enough fully-charged batteries to the BSS, it has no restricted requirements on the detailed amount of energy exported at each period in the operation horizon of the nanogrid. Therefore, this agreement can be seen as a dispatchable load in balancing the variable PV generation and demands in the off-grid nanogrid.

For comparison, Fig. 11 gives the corresponding cases that the nanogrid exports no batteries to the BSS. In this case, the BESS in the nanogrid keeps charging till it is fully charged at Hour 12. Then, in the following hours, the nanogrid has to curtail a considerable part $(43.4 \%)$ of the costly PV generation. This inevitably leads to underutilization of the costly PV and BESS resources.

\subsection{Computational issues}

All simulations were carried out using CPLEX 12.1 under the MATLAB interface YALMIP on a PC with Intel i7 $2.8 \mathrm{GHz}$ processor (8 GB RAM). Each MILP computation requires $25 \mathrm{~s}$ to $70 \mathrm{~s}$, and it took a little more than 72 min to obtain all the solutions. The computational requirements would grow when the number of uncertain parameters increases. However, it should not be an issue since the simulation is performed off-line in the design stage, and parallel computing technique can be employed to speed up the solutions.

\section{Conclusions and future work}

This paper presents an RO approach to determine the optimal mix of PV generation and BESS in an off-grid nanogrid, which powers its local loads and supplies fullycharged batteries to a BSS. The employed RO approach uses distribution-free bounded intervals to model uncertainties of PV generation and demands. It can control the robustness of the capacity planning results by flexibly adjusting the predefined uncertainty budgets. The results of case studies show that a nanogrid-based BSS framework has potentials to offer economic benefits, ensure reliability, and prevent underutilization of capital-intensive PV and BESS.

Swapping batteries between a BSS and a nanogrid can achieve mutual benefits, and it is a potential approach to facilitating higher penetration levels of distributed PV and catalyzing the popularity of EVs. Future studies will focus on developing flexible energy management strategies for nanogrids and expanding the role of the BSS in coordinating various distributed energy resources.

Acknowledgments This work was jointly supported by the National Natural Science Foundation of China (No. 51377035) and the ChinaUK NSFC/EPSRC EV project (No. 51361130153). The authors also would like to thank the anonymous reviewers and the editor for their valuable comments.

Open Access This article is distributed under the terms of the Creative Commons Attribution 4.0 International License (http:// creativecommons.org/licenses/by/4.0/), which permits unrestricted use, distribution, and reproduction in any medium, provided you give appropriate credit to the original author(s) and the source, provide a link to the Creative Commons license, and indicate if changes were made.

\section{References}

[1] Shahidehpour M, Li Z, Gong W et al (2017) A hybrid AC/DC nanogrid: the keating hall installation at the Illinois Institute of Technology. IEEE Electrif Mag 5(2):36-46

[2] Werth A, Kitamura N, Tanaka K (2015) Conceptual study for open energy systems: distributed energy network using interconnected DC nanogrids. IEEE Trans Smart Grid 6(4):1621-1630

[3] Wang C, Yang X, Wu Z et al (2014) A highly integrated and reconfigurable microgrid testbed with hybrid distributed energy sources. IEEE Trans Smart Grid 7(1):451-459

[4] Wang S, Li Z, Wu L et al (2013) New metrics for assessing the reliability and economics of microgrids in distribution system. IEEE Trans Power Syst 28(3):2852-2861 
[5] Burmester D, Rayudu R, Seah W et al (2017) A review of nanogrid topologies and technologies. Renew Sustain Energy Rev 67:760-775

[6] Liu W, Niu S, Xu H (2017) Optimal planning of battery energy storage considering reliability benefit and operation strategy in active distribution system. J Mod Power Syst Clean Energy 5(2):177-186

[7] Liu W, Sun L, Lin Z et al (2016) Multi-objective restoration optimisation of power systems with battery energy storage systems. IET Gener Transm Distrib 10(7):1749-1757

[8] Guo L, Yu Z, Wang C et al (2016) Optimal design of battery energy storage system for a wind-diesel off-grid power system in a remote Canadian community. IET Gener Transm Distrib 10(3):608-616

[9] Liu N, Yu X, Fan W et al (2017) Online energy sharing for nanogrid clusters: a Lyapunov optimization approach. IEEE Trans Smart Grid. https://doi.org/10.1109/TSG.2017.2665634

[10] Liu W, Wu Q, Wen F et al (2014) Day-ahead congestion management in distribution systems through household demand response and distribution congestion prices. IEEE Trans Smart Grid 5(6):2739-2747

[11] Tushar W, Chai B, Yuen C et al (2016) Energy storage sharing in smart grid: a modified auction based approach. IEEE Trans Smart Grid 7(3):1462-1475

[12] Ban M, Shahidehpour M, Yu J et al (2017) A cyber-physical energy management system and optimal sizing of networked nanogrids with battery swapping stations. IEEE Trans Sustain Energy. https://doi.org/10.1109/TSTE.2017.2788056

[13] Jing ZX, Zhu JZ, Hu RX (2018) Sizing optimization for island microgrid with pumped storage system considering demand response. J Mod Power Syst Clean Energy 6(4):791-801

[14] Martinez IJ, Garcia-villalobos J, Zamor I et al (2017) Energy management of micro renewable energy source and electric vehicles at home level. J Mod Power Syst Clean Energy 5(6):979-990

[15] Yao W, Chung C, Wen F et al (2016) Scenario-based comprehensive expansion planning for distribution systems considering integration of plug-in electric vehicles. IEEE Trans Power Syst 31(1):317-328

[16] Zhu X, Yan J, Lu N (2017) A graphical performance-based energy storage capacity sizing method for high solar penetration residential feeders. IEEE Trans Smart Grid 8(1):3-12

[17] Zhang Y, Ren S, Dong ZY et al (2017) Optimal placement of battery energy storage in distribution networks considering conservation voltage reduction and stochastic load composition. IET Gener Transm Distrib 11(15):3862-3870

[18] Wang C, Jiao B, Guo L et al (2014) Optimal planning of standalone microgrids incorporating reliability. J Mod Power Syst Clean Energy 2(3):195-205

[19] Hajipour E, Bozorg M, Fotuhi-Firuzabad M (2015) Stochastic capacity expansion planning of remote microgrids with wind farms and energy storage. IEEE Trans Sustain Energy $6(2): 491-498$

[20] Zhao J, Wang J, Xu Z et al (2017) Distribution network electric vehicle hosting capacity maximization : a chargeable region optimization model. IEEE Trans Smart Grid 32(5):4119-4130

[21] Wang S, Dong Z, Luo F et al (2018) Stochastic collaborative planning of electric vehicle charging stations and power distribution system. IEEE Trans Ind Inform 14(1):321-331

[22] Wang Z, Chen B, Wang J et al (2014) Robust optimization based optimal DG placement in microgrids. IEEE Trans Smart Grid 5(5):2173-2182

[23] Khodaei A, Bahramirad S, Shahidehpour M (2015) Microgrid planning under uncertainty. IEEE Trans Power Syst 30(5):2417-2425
[24] Jabr R, Džafić I, Pal B (2015) Robust optimization of storage investment on transmission networks. IEEE Trans Power Syst 30(1):531-539

[25] Zhang C, Xu Y, Dong Z et al (2017) Robust operation of microgrids via two-stage coordinated energy storage and direct load control. IEEE Trans Power Syst 32(4):2858-2868

[26] Bertsimas D, Sim M (2004) The price of robustness. Oper Res 52(1):35-53

[27] Li J, Wen J, Han X (2015) Low-carbon unit commitment with intensive wind power generation and carbon capture power plant. J Mod Power Syst Clean Energy 3(1):63-71

[28] Mejía-Giraldo D, McCalley J (2014) Adjustable decisions for reducing the price of robustness of capacity expansion planning. IEEE Trans Power Syst 29(4):1573-1582

[29] Wang C, Zhou Y, Wu J et al (2015) Robust-index method for household load scheduling considering uncertainties of customer behavior. IEEE Trans Smart Grid 6(4):1806-1818

[30] Ni L, Wen F, Liu W et al (2017) Congestion management with demand response considering uncertainties of distributed generation outputs and market prices. J Mod Power Syst Clean Energy 5(1):66-78

[31] Das R, Madani V, Aminifar F et al (2015) Distribution automation strategies: evolution of technologies and the business case. IEEE Trans Smart Grid 6(4):2166-2175

[32] Liu N, Chen Z, Liu J et al (2014) Multi-objective optimization for component capacity of the photovoltaic-based battery switch stations: towards benefits of economy and environment. Energy 64:779-792

[33] Yao L, Yang B, Cui H et al (2016) Challenges and progresses of energy storage technology and its application in power systems. J Mod Power Syst Clean Energy 4(4):519-528

[34] Savkin AV, Khalid M, Agelidis VG (2016) A constrained monotonic charging/discharging strategy for optimal capacity of battery energy storage supporting wind farms. IEEE Trans Sustain Energy 7(3):1224-1231

[35] Wu S, Xu Q, Li Q et al (2017) An optimal charging strategy for PV-based battery swapping stations in a DC distribution system. Int J Photoenergy 2017:1-11

[36] Yang Z, Li K, Niu Q et al (2017) A comprehensive study of economic unit commitment of power systems integrating various renewable generations and plug-in electric vehicles. Energy Convers Manag 132:460-481

[37] Simoglou C, Biskas P, Bakirtzis A (2010) Optimal selfscheduling of a thermal producer in short-term electricity markets by MILP. IEEE Trans Power Syst 25(4):1965-1977

[38] Morales-españa G, Latorre J, Ramos A (2013) Tight and compact MILP formulation for the thermal unit commitment problem. IEEE Trans Power Syst 28(4):4897-4908

[39] Li T, Shahidehpour M (2005) Price-based unit commitment: a case of lagrangian constants. IEEE Trans Power Syst 20(4):2015-2025

[40] Abreu L, Khodayar M, Shahidehpour M et al (2012) Riskconstrained coordination of cascaded hydro units with variable wind power generation. IEEE Trans Sustain Energy 3(3):359-368

[41] Wang G, Zhao J, Wen F et al (2015) Dispatch strategy of PHEVs to mitigate selected patterns of seasonally varying outputs from renewable generation. IEEE Trans Smart Grid 6(2):627-639

Mingfei BAN received the B.S. and M.S. degrees in electrical engineering from Harbin Institute of Technology, Harbin, China, in 2011 and 2013, respectively, where he is currently working toward the Ph.D. degree. He is a visiting Ph.D. student at Illinois Institute of 
Technology (IIT). His research interests include smart grid and electrical vehicles.

Jilai YU is currently a Professor and the director of Electric Power Research Institute at Harbin Institute of Technology. His current research interests include power system analysis and control, optimal dispatch of power system, green power and smart grid.

Mohammad SHAHIDEHPOUR is the Bodine Distinguished Professor and Chair in the Electrical and Computer Engineering Department at Illinois Institute of Technology (IIT), Chicago. He is also a Research Professor at the King Abdulaziz University in Saudi Arabia. Dr. Shahidehpour is a member of the US National Academy of Engineering. His current research interests include power system operation and planning, microgrids and energy hubs, sustainable energy integration.

Danyang GUO received the M.S. degree in the Electrical Department at Xi' an Jiaotong University in 2014. She is working toward the Ph.D. degree in electrical engineering in Harbin Institute of Technology. Her research interests include economic dispatch and the environmental generating. 